ARTICLE

https://doi.org/10.1038/s41467-020-14472-0

\title{
Valley phonons and exciton complexes in a monolayer semiconductor
}

Minhao He (1) 10, Pasqual Rivera (1) 1,10, Dinh Van Tuan², Nathan P. Wilson', Min Yang (1) 2, Takashi Taniguchi Kenji Watanabe (i) ${ }^{3}$, Jiaqiang Yan ${ }^{4,5}$, David G. Mandrus ${ }^{4,5,6}$, Hongyi Yu (1) ${ }^{7}$, Hanan Dery ${ }^{2,8 \star}$, Wang Yao (1) ${ }^{7 \star} \&$ Xiaodong Xu (i) ${ }^{1,9 *}$

The coupling between spin, charge, and lattice degrees of freedom plays an important role in a wide range of fundamental phenomena. Monolayer semiconducting transitional metal dichalcogenides have emerged as an outstanding platform for studying these coupling effects. Here, we report the observation of multiple valley phonons - phonons with momentum vectors pointing to the corners of the hexagonal Brillouin zone - and the resulting exciton complexes in the monolayer semiconductor $\mathrm{WSe}_{2}$. We find that these valley phonons lead to efficient intervalley scattering of quasi particles in both exciton formation and relaxation. This leads to a series of photoluminescence peaks as valley phonon replicas of dark trions. Using identified valley phonons, we also uncover an intervalley exciton near charge neutrality. Our work not only identifies a number of previously unknown 2D excitonic species, but also shows that monolayer $\mathrm{WSe}_{2}$ is a prime candidate for studying interactions between spin, pseudospin, and zone-edge phonons.

\footnotetext{
${ }^{1}$ Department of Physics, University of Washington, Seattle, WA 98195, USA. ${ }^{2}$ Department of Electrical and Computer Engineering, University of Rochester, Rochester, NY 14627, USA. ${ }^{3}$ National Institute for Materials Science, Tsukuba, Ibaraki 305-0044, Japan. ${ }^{4}$ Materials Science and Technology Division, Oak Ridge National Laboratory, Oak Ridge, TN 37831, USA. ${ }^{5}$ Department of Materials Science and Engineering, University of Tennessee, Knoxville, TN 37996, USA. ${ }^{6}$ Department of Physics and Astronomy, University of Tennessee, Knoxville, TN 37996, USA. ${ }^{7}$ Department of Physics and Center of Theoretical and Computational Physics, University of Hong Kong, Hong Kong, China. ${ }^{8}$ Department of Physics and Astronomy, University of Rochester, Rochester, NY 14627 , USA. ${ }^{9}$ Department of Materials Science and Engineering, University of Washington, Seattle, WA 98195, USA. ${ }^{10}$ These authors contributed equally: Minhao He, Pasqual Rivera. *email: hanan.dery@rochester.edu; wangyao@hku.hk; xuxd@uw.edu
} 
lectron-phonon interaction is a ubiquitous process in solids. In monolayer semiconducting transition metal dichalcogenides (TMDs), the broken inversion symmetry and strong spin-orbit coupling leads to the well-known spin-valley coupling of band edge electrons ${ }^{1-6}$. The emergent valley-contrasting properties not only impact quasiparticles, but also are expected to give rise to new physics involving zone edge phonons, or valley phonons, which are collective lattice oscillations at the corners of hexagonal Brillouin zone ( $\pm K$ points). These phonons have been predicted to play an important role in spin and valley pseudospin relaxation through phonon-assisted intervalley scattering ${ }^{7,8}$. Additionally, valley phonons can possess chirality with intrinsic pseudo-angular momentum ${ }^{9}$, which has recently attracted wide attention ${ }^{10-13}$. Such chiral phonons have nontrivial Berry curvature and are predicted to give rise to valley phonon Hall effect, a counterpart of valley Hall effect of electrons in 2D semiconductors ${ }^{14-16}$. Despite the importance of valley phonons, experimental progress in understanding their properties has been limited, since it is challenging to probe phonons with large momentum vectors.

Here, we identify the signatures of multiple valley phonons in monolayer $\mathrm{WSe}_{2}$. The monolayer $\mathrm{WSe}_{2}$ hosts stable and long-lived dark exciton and trion as ground states ${ }^{17-22}$. The resulting accumulated exciton and trion populations are highly desirable for studying their interactions with phonons. We found that three valley phonons facilitate efficient spin-conserving intervalley scattering, which results in a series of dark exciton and dark trion phonon-replicas in the low-temperature photoluminescence (PL) spectrum. The sign and magnitude of Landé effective $g$-factors of the various replicas, together with their PL helicity under optical pumping, reveals that the spin-preserving intervalley scattering of the electron is more efficient than its intravalley spin-flip during the dark exciton/trion formation process. This results in a surprising finding: the single electron in both the positive dark trion and intervalley exciton resides in the valley opposite to that which the optical pump is coupled to. Moreover, the identified intervalley exciton resonance enables us to infer a short-range electron-hole exchange interaction of $\sim 10 \mathrm{meV}$ by extracting the energy splitting between intervalley and dark (intravalley) excitons.

\section{Results}

Gate-dependent PL spectrum of monolayer $\mathrm{WSe}_{2}$. The samples are exfoliated monolayer $\mathrm{WSe}_{2}$ encapsulated between thin flakes of hexagonal boron nitride (hBN). Few-layered graphene serves as a local bottom gate for electrostatic control of the monolayer carrier density (see the "Methods" section). Figure $1 \mathrm{a}, \mathrm{b}$ are an optical microscope image and schematic of a representative device, respectively. Figure $1 \mathrm{c}$ shows the PL intensity plot as a function of gate voltage $(V)$ and photon energy, at a temperature of $1.6 \mathrm{~K}$. The laser energy is $1.775 \mathrm{eV}$ with right circularly polarized $\left(\sigma^{+}\right)$excitation and unpolarized detection. The full helicityresolved gate-dependent spectra are shown in Supplementary Fig. 1. Monolayer $\mathrm{WSe}_{2}$ hosts a rich spectrum of excitonic species $^{23-28}$. Several previously identified excitonic states are indicated in the figure, including the neutral bright exciton, bright trions ${ }^{29-33}$, the intravalley spin-forbidden dark exciton ${ }^{17-20,34}$ and dark trions ${ }^{18,35}$. The recently identified zone-center $\Gamma_{5}$ - or $E^{\prime \prime}-$ phonon replicas below both neutral ${ }^{12,13}$ and charged dark excitons $^{12}$ are also resolved, as indicated by black arrows.

The focus of this paper is to understand the valley phonon origin of several previously unidentified PL peaks. In particular, we focus on the triplet and quadruplet PL peaks outlined by dashed black boxes, and those pointed at by the white arrows in Fig. 1c. We have measured multiple samples that exhibit similar spectrum (Supplementary Fig. 2), and the nearly identical power dependence of the peaks of interest across several samples rules out the possibility of them arising due to defect states (Supplementary Fig. 3). As we explain below, all these peaks arise from the coupling of optically dark states to the bright ones via emitting valley phonons, which results in a series Stokesshifted valley phonon-replicas of the dark exciton and dark trions in the PL spectrum.

Valley phonon replicas of positively charged dark trion. We first consider the quadruplet PL peaks in the hole doping regime. Figure 2a shows the circular polarization resolved PL with $\sigma^{+}$ polarized excitation. The photon energy is relative to the positive dark trion $\left(D^{+}\right)$, and the intensity of the quadruplets is multiplied a

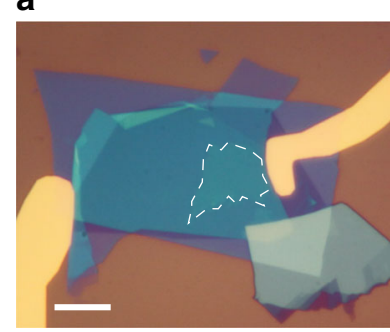

b

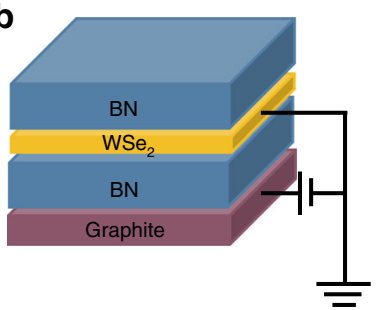

C

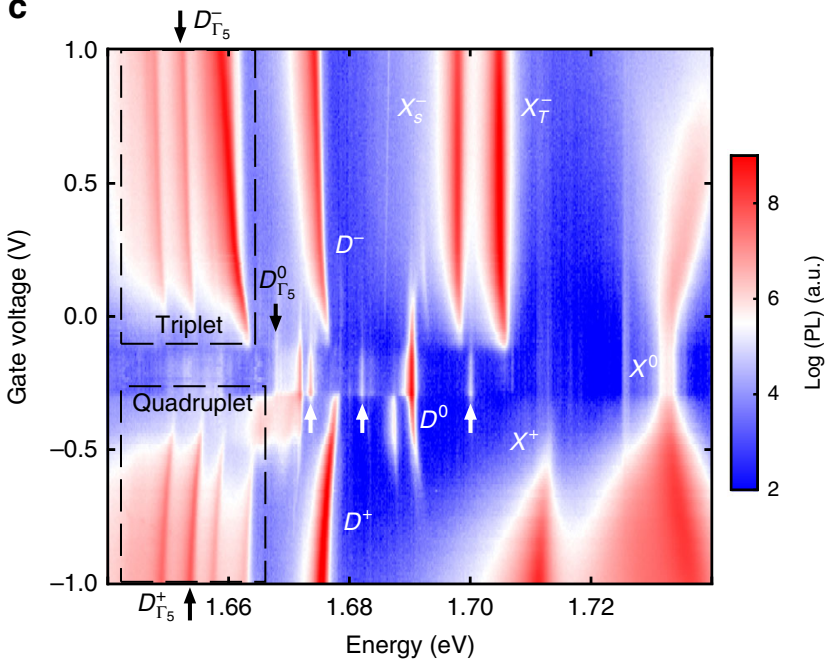

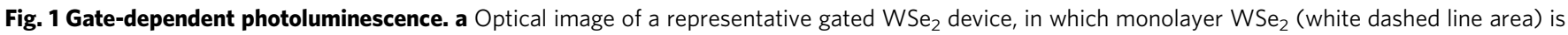

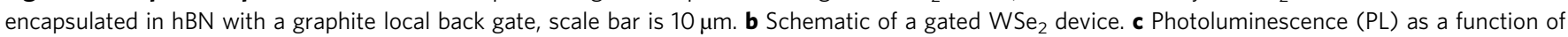
back gate voltage and photon energy. Excitonic states which have been reported in the literature are identified and marked. $X^{0}$ : neutral exciton; $X^{+}$: positive trion; $X_{\mathrm{S}}^{-}$and $X_{\mathrm{T}}^{-}$: intravalley and intervalley trion; $D^{0}$ : spin forbidden neutral dark exciton; $D^{+}$: positive dark trion; $D^{-}$negative dark trion. The unidentified triplet PL peaks at electron doping, quadruplet PL peaks at hole doping, and three states at the neutral regime, pointed to by the white arrows, are the focus of this work. 
a

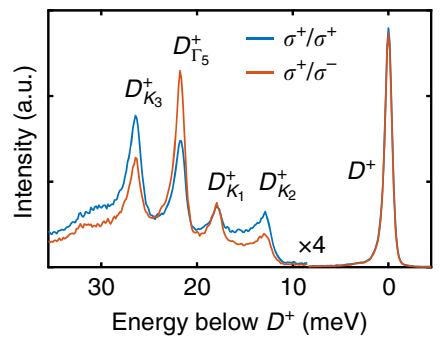

b

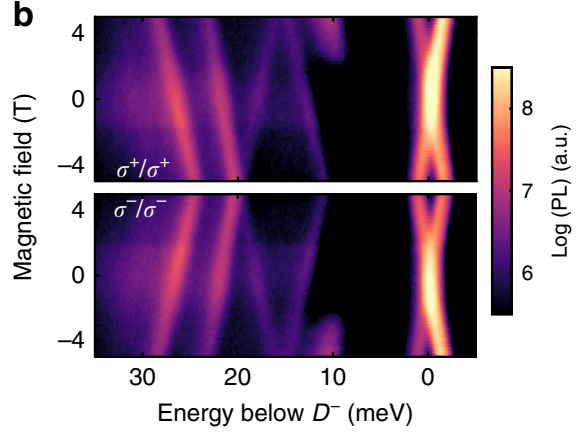

C

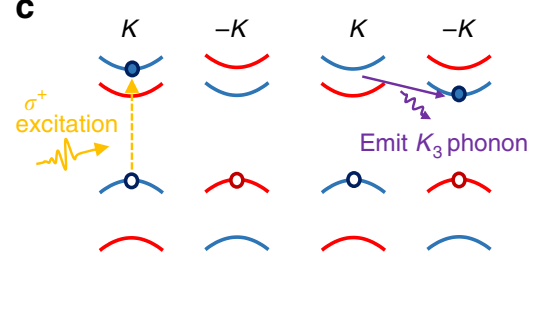

e

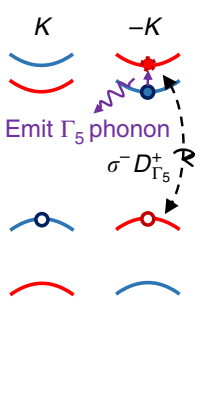

f

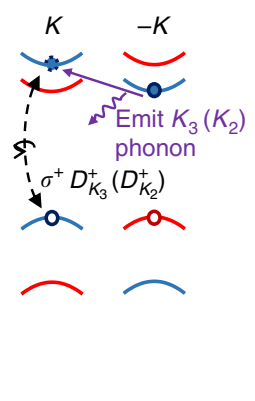

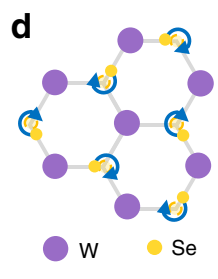

$K_{3}$ phonon

g

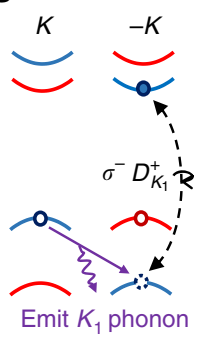

Fig. 2 Valley phonon replicas of positively charged dark trion. a Circular polarization resolved PL of the quadruplet PL peaks on the hole doping side. The photon energy of the spectrum is offset with respect to the positively charged dark trion $\left(D^{+}\right)$. The intensity of the quadruplets is multiplied by a factor 4 to emphasize the weak spetrum features. b Circular polarization resolved magneto PL of the quadruplets with $\sigma^{+} / \sigma^{+}$(top) and $\sigma^{-} / \sigma^{-}$(bottom) excitation/ detection. c Schematic of $D^{+}$formation process under $\sigma^{+}$excitation. Blue and red represent bands with electron having spin up and down, respectively. Filled and unfilled circles represent electron and missing electron (hole) in the conduction and valence band, respectively. Orange dashed line indicates photo excitation. Purple arrow denotes the emission of valley phonon. $\mathbf{d}$ Illustration of vibrational normal mode of $K_{3}$ phonon, wherein Se atoms orbit around their equilibrium positions. See Supplementary Fig. 6 for other valley phonons. e- $\mathbf{g}$ Schematic of phonon-assisted emission process of $D_{\Gamma_{5}}^{+}$ $D_{\mathrm{K}_{3}}^{+}\left(D_{\mathrm{K}_{2}}^{+}\right)$, and $D_{\mathrm{K}_{1}}^{+}$phonon replicas, respectively. Dashed circle: virtual state. As indicated by the purple arrow, the optically dark state couples to the bright ones by emitting a valley phonon, resulting in a Stokes shift of the dark trion emission by the recombination of an electron-hole pair (black dashed line). See text for details.

by a factor of 4 to emphasize these weak spectral features. We ascribe the four peaks to bright replicas of the dark trion, mediated by interactions with phonons. The quadruplets are labeled as $D_{K_{3}}^{+}, D_{\Gamma_{5}}^{+}, D_{K_{1}}^{+}, D_{K_{2}}^{+}$, which correspond to their origin from coupling of $D^{+}$with valley phonons $K_{3}, K_{1}$, and $K_{2}$, and the zone-center phonon $\Gamma_{5}$. This nomenclature has its origin in the Koster notation of the $K$-point irreducible representations of the $C_{3 h}$ point double-group corresponding to the symmetry of the monolayer semiconductors (Supplementary Table 3, character table).

The valley phonon replicas $D_{K_{3}}^{+}, D_{K_{1}}^{+}, D_{K_{2}}^{+}$are 26,18 , and $13 \mathrm{meV}$ below $D^{+}$, respectively. The association with zone-edge phonons $K_{3}, K_{1}$, and $K_{2}$ is two-fold. The first reason is that the energy differences between $D^{+}$and its replicas match the energies of the associated phonon modes (Supplementary Fig. 5, phonon spectrum). The second reason is rooted in the selection rules of electron-phonon coupling; ${ }^{7}$ intervalley transitions of electrons and holes are mediated only by specific phonons (see discussions in Supplementary Notes 3 and 4). Finally, we note that $D_{\Gamma_{5}}^{+}$ appears $22 \mathrm{meV}$ below $D^{+}$, which is consistent with the recently reported $\Gamma_{5}$ phonon replica of $D^{+12}$.

To further examine these assignments, we performed magnetoPL to extract the effective Landé $g$-factors of the various states, which are then used to identify the spin and valley indices of the constituent electrons and holes in the excitonic states ${ }^{36-40}$. The extracted $g$-factors of the states of interest are listed in Table 1. The $g$-factors of other states are listed in Supplementary Table 1, and a detailed analysis can be found in Supplementary Note 2. Briefly, for intravalley electron-hole recombination, the magnitude of the $g$-factor is about 4 for parallel electron and hole spins ${ }^{36,37,39,40}$, and about 9 when the two spins are anti-parallel ${ }^{12,13,21}$. Meanwhile, if the recombination involves electron and hole from opposite valleys but with parallel spins, a value about 13 is expected.

Figure $2 \mathrm{~b}$ shows the circular polarization resolved PL intensity as a function of out-of-plane magnetic field. The energy scale is relative to the position of $D^{+}$at zero applied magnetic field. Both the cross pattern of $D^{+}$in Fig. $2 \mathrm{~b}$ and its unpolarized light emission (see Fig. 2a) result from the underlying out-of-plane dipole orientation ${ }^{12}$, and are hallmarks of direct intravalley recombination of dark trions. Following the convention of valley Zeeman splitting as $\Delta=E\left(\sigma^{+}\right)-E\left(\sigma^{-}\right)$, where $E\left(\sigma^{+}\right)$and $E\left(\sigma^{-}\right)$ are the peak energies of the $\sigma^{+}$and $\sigma^{-}$polarized PL components, we obtained $g\left(D^{+}\right)=-8.6$. The obtained $g$-factor of $D^{+}$is therefore consistent with expectations for the direct recombination through the intravalley spin-flip transition.

The Zeeman shifts of the quadruplet PL peaks underpin their origin as phonon replicas of the dark positive trion $D^{+}$. The extracted $g$-factors of $-13.4,12.2$, and -13.0 for $D_{K_{3}}^{+}, D_{K_{1}}^{+}, D_{K_{2}}^{+}$, respectively, are consistent with intervalley recombination of electron and hole. Note that the $g$ factor sign of $D_{K_{1}}^{+}$is opposite to others, which will be discussed later. While the intervalley recombination is naturally forbidden for delocalized exciton complexes, because of the large momentum mismatch, emission of a valley phonon can supply the required momentum, resulting in phonon-assisted luminescence of the otherwise dark states. Combined with the concurrence of similar valley phonon energies and energy difference between the peaks and $D^{+}$, we conclude that $D_{K_{3}}^{+}, D_{K_{1}}^{+}, D_{K_{2}}^{+}$are $K_{3}, K_{1}$, and $K_{2}$ valley phonon replicas of $D^{+}$.

The polarization of the $D_{\Gamma_{5}}^{+}$peak highlights the importance of valley phonons in the formation process of $D^{+}$. From Fig. 2a, we observe that $D_{\Gamma_{5}}^{+}$is cross-circularly polarized. The valley optical 
Table 1 Effective Landé $g$-factors of $\mathbf{W S e}_{2}$ dark excitonic states and their phonon replicas.

\begin{tabular}{lllll} 
& Dark state & $\boldsymbol{K}_{\mathbf{2}}$ replica & $\boldsymbol{K}_{\mathbf{1}}$ replica & $\boldsymbol{\Gamma}_{\mathbf{5}}$ replica \\
\hline Neutral regime: $I^{0}$ & -12.5 & - & 12.0 & - \\
Neutral regime: $D^{0}$ & -9.1 & - & - & -9.8 \\
Hole doped: $D^{+}$ & -8.6 & -13.0 & 12.2 & -12.6 \\
Electron doped: $D^{-}$ & -9.5 & -13.6 & 12.2 & -9.7 \\
\hline
\end{tabular}

selection rules dictate that $\sigma^{+}$excitation creates an electron and hole in the $+K$ valley, while $\sigma^{-}$polarized emission can only happen through spin-conserved electron-hole recombination in the $-K$ valley. In addition, $g\left(D_{\Gamma_{5}}^{+}\right)=-9.7$ indicates the intravalley electron-hole recombination nature of the peak. The cross-polarized emission of $D_{\Gamma_{5}}^{+}$therefore leads to the surprising conclusion that $\sigma^{+}$excitation results in $D^{+}$with the single electron located in the $-K$ valley: i.e. $D^{+}(-K)$.

The cross polarization of $D^{+}$can be understood by considering the impact of phonons on the electron relaxation pathways following photoexcitation. The $D^{+}$population is created by optical pumping of the spin-conserved interband transition (Fig. 2c), followed by the relaxation of the electron from the higher energy spin-valley locked sub-band to the lower energy one. The latter step requires either a spin-flip or a valley-flip. From symmetry analysis, the $\Gamma_{5}$ phonon can lead to the intravalley spin-flip relaxation of electron from upper to lower conduction band 7 , but it cannot cause intervalley scattering. On the other hand, spin-conserving intervalley scattering of the electron with $K_{3}$ phonon is a symmetry-allowed zeroth-order channel $^{8}$. Figure $2 c$ illustrates the intervalley electron-phonon relaxation process that leads to the formation of $D^{+}(-K)$. First, $\sigma^{+}$excitation creates electron in the spin up conduction band in the $K$ valley. Assisted by the $K_{3}$ valley phonon (Fig. 2 d), this spin up electron is then scattered into the spin up band in the $-K$ valley, forming $D^{+}(-K)$ with two holes separately located at the top of $\pm K$ valleys. $D^{+}(-K)$ then couples to $\sigma^{-}$polarized photon by emitting $\Gamma_{5}$ phonon, as shown in Fig. 2e. Evidently, the observed cross-polarized $D_{\Gamma_{5}}^{+}$emission implies that the valley-flip rate exceeds the spin-flip one in the relaxation of electron.

Having established that $\sigma^{+}$polarized excitation results in $D^{+}(-K)$, the understanding of both $D_{K_{3}}^{+}$and $D_{K_{2}}^{+}$is straightforward. As indicated in Fig. $2 \mathrm{f}$, the spin up electron in the lower $-K$ sub-band is virtually scattered to the higher $+K$ sub-band by emitting either a $K_{3}$ or $K_{2}$ valley phonon (see Supplementary Note 5 for further analysis of $K_{2}$ ). The spin-conserving intervalley scatter then allows for recombination with the hole in the $+K$ valley, emitting $\sigma^{+}$polarized photon with energy either $26 \mathrm{meV}$ $\left(K_{3}\right)$ or $13 \mathrm{meV}\left(K_{2}\right)$ below $\mathrm{D}^{+}$. The $g$-factors of $D_{K_{3}}^{+}$and $D_{K_{2}}^{+}$are nearly equal, about -13.4 and -13.0 , respectively, and correspond to expected values for the intervalley spin-conserving electron-hole recombination.

Moreover, the measured amplitude of $D_{K_{3}}^{+}$, which is several times stronger than that of $D_{K_{2}}^{+}$, is also consistent with grouptheory selection rules. In particular, the selection rules dictate that, in pristine monolayer $\mathrm{WSe}_{2}$, only the $K_{3}$ phonon mode can induce intervalley electron transitions between conduction band edges at the high symmetry $K$ and $-K$ points ${ }^{7}$. Meanwhile, intervalley electron transitions that are mediated by other $K$-point phonon modes are higher-order processes with correspondingly smaller amplitudes that involve electron states in the neighborhood of $\pm K$. Such relatively weak processes can be amplified by several possible sources, such as localization next to defects, breaking $\mathrm{WSe}_{2}$ mirror inversion symmetry by $\mathrm{hBN}$ encapsulation, or $\mathrm{WSe}_{2} / \mathrm{hBN}$ moire superlattice providing in plane momentum.

The positive $g$-factor of $D_{\mathrm{K}_{1}}^{+}$is a signature of the interaction of the hole and $K_{1}$ valley phonon. According to the group-theory selection rules, $K_{1}$ is the only phonon mode that enables the spinconserving intervalley transition between valence-band states at $K$ and $-K$ points (i.e., it is a zeroth-order process) ${ }^{7}$. As shown in Fig. $2 \mathrm{~g}$, the hole in the higher valence band of $K$ valley is virtually scattered to the lower valence band of the $-K$ valley with the same spin orientation via emission of a $K_{1}$ valley phonon, forming an intermediate virtual $\mathrm{B}$ trion. The recombination of the electron with this scattered hole in the $-K$ valley results in $D_{K_{1}}^{+}$, with energy $18 \mathrm{meV}$ below $D^{+}$. While the initial and final states have the same spin-valley configuration as in $D_{K_{3}}^{+}$and $D_{K_{2}}^{+}$, the emission from $D_{K_{1}}^{+}$is $\sigma^{-}$polarized. Therefore, the $g$-factor of $D_{K_{1}}^{+}$is expected to have similar magnitude, but opposite sign, compared to the $g$-factors of $D_{K_{3}}^{+}$and $D_{K_{2}}^{+}$, as we have observed. Note that although the interaction between the hole and valley phonon $K_{1}$ is relatively strong, the coupling of $D^{+}(-K)$ (or A trion) with the virtual B trion state is $\sim 400 \mathrm{meV}$ detuned, which is much larger than in the case of $K_{3}$ intervalley electron scattering. Therefore, the PL intensity of $D_{K_{1}}^{+}$is expected to be several times weaker than that of $D_{K_{3}}^{+}$, which is in agreement with our observation (see Supplementary Note 4).

Valley phonon replicas of negatively charged dark trion. The valley phonon replicas of the negative dark trion $D^{-}$can be understood using similar analysis as above. Figure 3a shows the helicity-resolved PL under $\sigma^{+}$polarized excitation. The lower energy spectral features, denoted as $T_{1}, D_{K_{3}}^{-}$, and $D_{\Gamma_{5}}^{-}$show appreciable co-circular polarization. Figure $3 \mathrm{~b}$ shows the PL intensity as a function of magnetic field and photon energy, with $\sigma^{-} / \sigma^{-}$polarized excitation/detection (the $\sigma^{+} / \sigma^{+}$results are shown in Supplementary Fig. 7). The extracted $g$-factor of $D_{K_{3}}^{-}$ is -12.5 , indicating intervalley recombination, and the energy is $26 \mathrm{meV}$ below $D^{-}$. As such, we can identify that $D_{K_{3}}^{-}$originates from the interaction of $-K$ valley electron with $K_{3}$ phonon, as depicted in the inset of Fig. 3a. The $g$-factor of $D_{\Gamma_{5}}^{-}(-9.9)$ is nearly the same as that of $D^{-}(-9.5)$, and as expected, the peak appears $22 \mathrm{meV}$ below $D^{-}$.

The observation of triplet peak pattern in the PL spectrum under electron doping is slightly different from the quadruplet one observed under hole doping. Since $T_{1}$ is more intense than $D^{-}$, and its $g$-factor is about -4.5 , it is not likely to be a phonon replica of $D^{-}$. Its origin is unknown, but $T_{1}$ is near the spectral range of the $K_{2}$ and $K_{1}$ valley phonon replicas, and thus obscures them entirely. Nevertheless, the large difference in $g$-factor of $T_{1}$ from that of $D^{-}$allows us to identify $D_{K_{2}}^{-}$in magneto-PL, since the states shift away from one another. We note that there is a faint line in Fig. 3b, appearing on the high energy shoulder of $T_{1}$ at high magnetic field. We ascribe this peak to $D_{K_{2}}^{-}$based on 
a
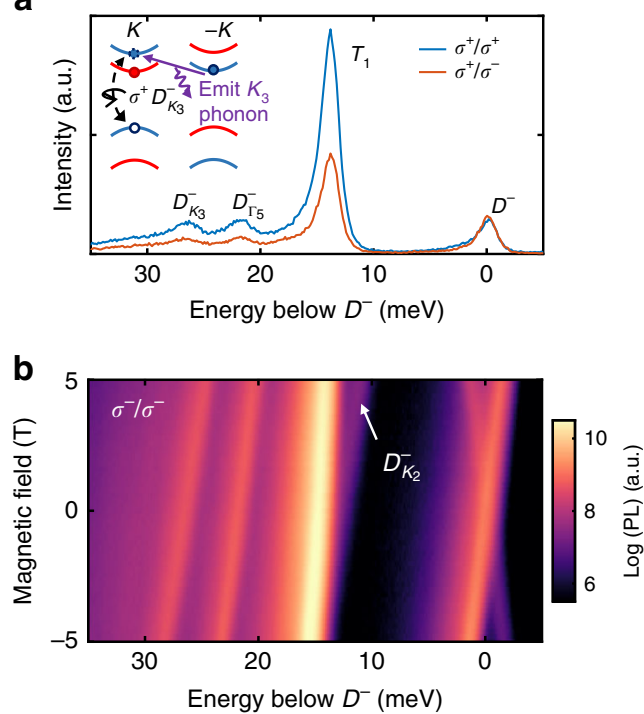
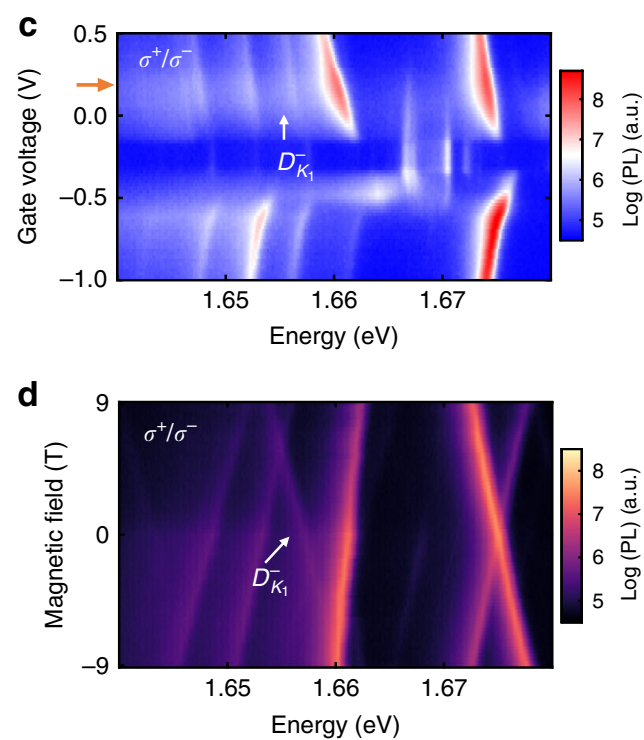

Fig. 3 Valley phonon replicas of negatively charged dark trion. a Circular polarization resolved PL of the triplet PL peaks under electron-doped conditions. The photon energy of the spectrum is offset with respect to the negatively charged dark trion ( $\left.D^{-}\right)$. $\mathbf{b} \mathrm{Magneto}^{\mathrm{PL}}$ of the triplets with $\sigma^{-}$excitation and copolarized detection. The photon energy of the spectrum is offset with respect to $D^{-}$at zero field. c Gate-dependent PL with $\sigma^{+}$resonant pumping of the bright exciton and cross polarized $\left(\sigma^{-}\right)$detection. The $D_{K_{1}}^{-}$state on the electron doping side, which is 18 meV below $D^{-}$, is clearly resolved. $\mathbf{d}$ Magneto $\mathrm{PL}$ at the gate voltage indicated by the orange arrow in $\mathbf{c}$, with $\sigma^{+}$excitation and $\sigma^{-}$detection.

its $g$-factor of about -13.6 , and its energy difference from $D^{-}$of $13 \mathrm{meV}$, both of which are in good agreement with expectations for $K_{2}$ phonon replica.

Meanwhile, $D_{K_{1}}^{-}$becomes evident when the excitation laser is resonant with the bright neutral exciton $\left(X^{0}\right)$. Figure $3 \mathrm{c}$ presents the PL intensity as a function of gate voltage and photon energy, with $\sigma^{+}$polarized excitation laser in resonance with $X^{0}(1.733 \mathrm{eV})$ and $\sigma^{-}$polarized detection. For $V=0.2 \mathrm{~V}$, arrow on side of Fig. $3 \mathrm{c}$, $D_{K_{1}}^{-}$becomes apparent at $18 \mathrm{meV}$ below $D^{-}$. Figure $3 \mathrm{~d}$ shows magneto-PL with the same cross-polarized polarization $\left(\sigma^{+} / \sigma^{-}\right)$ (see Supplementary Fig. 8 for complete data set). We find that $D_{K_{1}}^{-}$has a positive $g$-factor of 12.2 , which is identical to that of $D_{K_{1}}^{+}$. Therefore, despite the initial appearance, the four phonon replica states observed under hole-doping also appear under electron-doping conditions. Moreover, we observe similar magnitude and sign of $g$-factors, as well as energy separation from the parent dark trion state.

Identification of intervalley dark excitons and its valley phonon replica. The valley phonon-assisted momentum relaxation mechanism also produces phonon replicas for neutral dark excitons. Figure $4 \mathrm{a}$ shows the helicity-resolved PL spectra near charge neutrality $(V=-0.2 \mathrm{~V})$. The energy axis is offset from $I^{0}$, which is the sharp line $32 \mathrm{meV}$ below the bright neutral exciton in Fig. 1c. We also observe strong direct recombination from the neutral intravalley dark exciton $D^{0}$, at $42 \mathrm{meV}$ below $X^{0}$. We confirm that $D^{0}$ has negligible circular polarization, resulting from its out-of-plane dipole orientation, and the extracted $g$ factor $(-9.1)$ is in good agreement with previously reported values $^{12,13,21}$. Magneto-PL measurements with linear excitation $(V)$ and $\sigma^{+} / \sigma^{-}$collection are shown in the top/bottom panel of Fig. $4 \mathrm{~b}$, respectively. The observed PL peak at $22 \mathrm{meV}$ below $D^{0}$, with a $g$-factor of -9.8 is consistent with the reported $\Gamma_{5}$ phonon replica $D_{\Gamma_{5}}^{0} 12,13$. We further note that $D_{\Gamma_{5}}^{0}$ shows a zero-field splitting of $0.6 \mathrm{meV}$, which arises from the fine structure of $D^{0}$ (Supplementary Fig. 9) ${ }^{8,21}$. We note that there are two replicas of $D^{0}$ that are apparent in the magneto-PL (cross-patterns at 3 and $13 \mathrm{meV}$ below $D^{0}$ ). The peak position of the latter one matches that of positive dark trion thus is precursor of $\mathrm{D}^{+}$, while the former one cannot be unambiguously identified at this time.

The $I^{0}$ emission is distinct from that of $D^{0}$ and exhibits all the expected behavior of the momentum indirect, or intervalley dark exciton, which has not been previously identified in this system. This emission peak is strongly co-circularly polarized, with near unity polarization. Its $g$-factor of about -12.5 corresponds to the recombination of electron and hole residing in opposite valleys, implying that $I^{0}$ is the direct recombination of intervalley exciton. The formation of $I^{0}$ is a consequence of the fast valley-flip of electrons via scattering with $K_{3}$ phonon in the dark exciton formation, as illustrated in Fig. 4c. This is consistent with formation of $D^{+}(-K)$ discussed above (Fig. 2c). The $10 \mathrm{meV}$ energy splitting between $D^{0}$ and $I^{0}$ is then a direct measure of the short-range electron-hole exchange interactions ${ }^{41}$. We note that the direct photon emission from the momentum indirect $I^{0}$ is weak in intensity, even when compared to the spin-forbidden dark state $D^{0}$. However, weak PL from indirect states is not unprecedented. Similar to the case of indirect band-gap semiconductors such as silicon, recombination of intervalley excitons without phonons can be mediated by localization next to defects $^{42}$, which alleviates the need to conserve crystal momentum due to translation symmetry.

The assignment of $I^{0}$ is corroborated by the identifications of its $K_{1}$ and $K_{3}$ phonon replicas, made evident by examining the energy, polarization, and $g$-factors of the spectral features. The feature indicated as $I_{K_{1}}^{0}$ in Fig. 4a, is located $18 \mathrm{meV}$ below $I^{0}$, is crosscircularly polarized, and has a positive $g$-factor of 12.0. These values are nearly identical to those found for both $D_{K_{1}}^{+}$and $D_{K_{1}}^{-}$, and consistent with expectations for the $K_{1}$ valley phonon replica of $I^{0}$. The $I_{K_{1}}^{0}$ recombination process via intervalley hole scattering is illustrated in Fig. 4d. In addition, the peak $I_{K_{3}}^{0}$ is about $26 \mathrm{meV}$ below $I^{0}$ and has a $g$-factor of -12.6 , which is the same as $I^{0}$ and supports its origin as $K_{3}$ valley phonon replica of $I^{0}$ (Fig. 4e).

\section{Discussion}

In conclusion, we unravel the role of valley phonons in exciton and trion formation and their recombination in semiconducting 


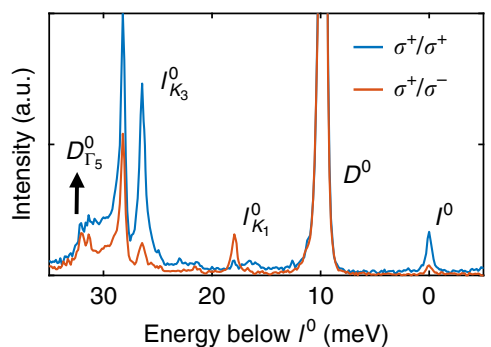

b

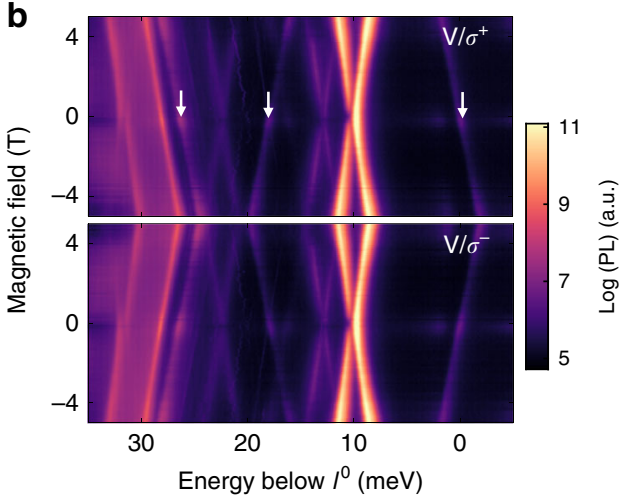

C

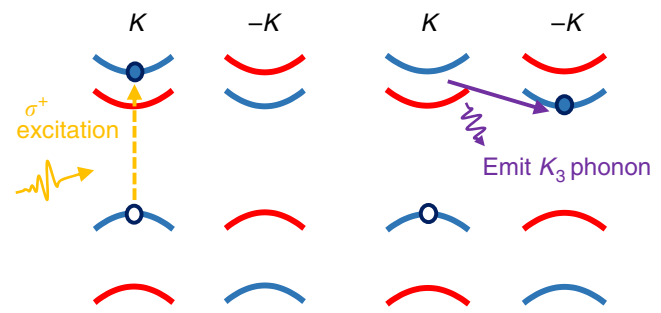

d

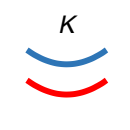

e

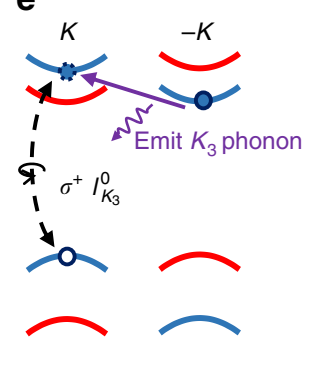

Fig. 4 Identification of intervalley dark exciton and its valley phonon replicas. a Circular polarization resolved PL in the neutral regime. The photon energy of the spectrum is offset with respect to the intervalley exciton $\left(I^{0}\right)$. b Magneto $\mathrm{PL}$ with linearly polarized excitation, $\sigma^{+}$(up) and $\sigma^{-}$polarized (down) detection. $I_{K_{3}}^{O}, I_{K_{1}}^{O}$ and $I^{O}$ are labeled by the white arrows, from left to right. c Schematic of intervalley exciton formation process under $\sigma^{+}$excitation, with assistance of $K_{3}$ phonon. $\mathbf{d}$, e Schematic of light emission process of $I_{K_{1}}^{0}$ (left) and $I_{K_{3}}^{0}$ (right) phonon replicas.

monolayer $\mathrm{WSe}_{2}$. Our work settles questions of the origin of nearly all of the observed peaks in the complex excitonic spectrum of monolayer $\mathrm{WSe}_{2}$. Another important result is that the relaxation of optically generated electrons from the upper conduction band to the lower conduction band is dominated by $K_{3}$ phonon-assisted spin-conserving intervalley scattering, rather than the $\Gamma_{5}$ phonon-assisted spin-flip intravalley scattering. Such a relaxation pathway gives rise to the unexpected initial state for the positively charged dark trion, and efficient formation of intervalley exciton $I^{0}$. This understanding is important for correct interpretation of excitonic spectral features, and may allow for new schemes to control the electron/exciton spin-valley state via optical pumping, e.g. coherent control of $D^{0}$ and $I^{0}$ populations via stimulated Raman adiabatic passage. Our work further motivates detailed studies of the electron-valley phonon coupling matrix elements by the first principle calculations, which should provide insights for theoretical models to gain a complete understanding of the complex monolayer $\mathrm{WSe}_{2}$ spectrum.

\footnotetext{
Methods

Sample fabrication. Monolayers of WSe $e_{2}$ were mechanically exfoliated from bulk crystals and identified by optical contrast, which was later confirmed by their lowtemperature PL spectrum. Thickness of hBN flakes used for encapsulation was typically $10-20 \mathrm{~nm}$, while the thickness of graphite back gate electrodes was typically around $5 \mathrm{~nm}$. Heterostructures of hBN/WSe2/hBN/Graphite are made with dry-transfer technique using polycarbonate films ${ }^{43}$. The surface of every flake in the heterostructure was confirmed clean with atomic force microscopy prior to fabrication. Finally, the V/Au contact are patterned with standard electron beam lithography and evaporation.
}

PL spectroscopy. PL measurements were performed with a confocal microscope in reflection geometry, with sample mounted in an exchange gas cooled cryostat (AttoDry 2100). The cryostat is equipped with a superconducting magnet in Faraday geometry (magnetic field $B$ perpendicular to sample plane). All measurements were performed at $1.6 \mathrm{~K}$ unless otherwise specified. A He-Ne laser $(632.8 \mathrm{~nm})$ or a frequency tunable continuous-wave Ti:sapphire laser were used to excite the sample. Polarization resolved PL measurements were performed with a set of broad-band half-wave plates, quarter wave plates and linear polarizers. PL signal was collected by a spectrometer with a silicon charge-coupled device.

\section{Data availability}

The data that support the findings of this study are available from the corresponding authors upon reasonable request.

Received: 13 December 2019; Accepted: 6 January 2020;

Published online: 30 January 2020

\section{References}

1. Xu, X. D., Yao, W., Xiao, D. \& Heinz, T. F. Spin and pseudospins in layered transition metal dichalcogenides. Nat. Phys. 10, 343-350 (2014).

2. Xiao, D., Liu, G.-B., Feng, W., Xu, X. \& Yao, W. Coupled spin and valley physics in monolayers of $\mathrm{MoS}_{2}$ and other group-VI dichalcogenides. Phys. Rev. Lett. 108, 196802 (2012).

3. Wang, G. et al. Colloquium: excitons in atomically thin transition metal dichalcogenides. Rev. Mod. Phys. 90, 021001 (2018).

4. Mak, K. F., Xiao, D. \& Shan, J. Light-valley interactions in 2D semiconductors Nat. Photon. 12, 451-460 (2018).

5. Cao, T. et al. Valley-selective circular dichroism of monolayer molybdenum disulphide. Nat. Commun. 3, 887 (2012).

6. Manzeli, S., Ovchinnikov, D., Pasquier, D., Yazyev, O. V. \& Kis, A. 2D transition metal dichalcogenides. Nat. Rev. Mater. 2, 17033 (2017).

7. Song, Y. \& Dery, H. Transport theory of monolayer transition-metal dichalcogenides through symmetry. Phys. Rev. Lett. 111, 026601 (2013).

8. Dery, H. \& Song, Y. Polarization analysis of excitons in monolayer and bilayer transition-metal dichalcogenides. Phys. Rev. B 92, 125431 (2015).

9. Zhang, L. \& Niu, Q. Chiral phonons at high-symmetry points in monolayer hexagonal lattices. Phys. Rev. Lett. 115, 115502 (2015).

10. Zhu, H. et al. Observation of chiral phonons. Science 359, 579-582 (2018).

11. Chen, $X$. et al. Entanglement of single-photons and chiral phonons in atomically thin $\mathrm{WSe}_{2}$. Nat. Phys. 15, 221-227 (2019).

12. Liu, E. et al. Valley-selective chiral phonon replicas of dark excitons and trions in monolayer WSe 2 . Phys. Rev. Research 1, 032007(R) (2019).

13. Li, Z. et al. Emerging photoluminescence from the dark-exciton phonon replica in monolayer WSe $\mathrm{W}_{2}$. Nat. Commun. 10, 2469 (2019).

14. Xiao, D., Yao, W. \& Niu, Q. Valley-contrasting physics in graphene: magnetic moment and topological transport. Phys. Rev. Lett. 99, 236809 (2007). 
15. Mak, K. F., McGill, K. L., Park, J. \& McEuen, P. L. The valley Hall effect in $\mathrm{MoS}_{2}$ transistors. Science 344, 1489-1492 (2014).

16. Gorbachev, R. V. et al. Detecting topological currents in graphene superlattices. Science 346, 448-451 (2014).

17. Zhang, X.-X., You, Y., Zhao, S. Y. F. \& Heinz, T. F. Experimental evidence for dark excitons in monolayer WSe ${ }_{2}$. Phys. Rev. Lett. 115, 257403 (2015).

18. Zhang, X.-X. et al. Magnetic brightening and control of dark excitons in monolayer $\mathrm{WSe}_{2}$. Nat. Nanotech. 12, 883 (2017).

19. Zhou, Y. et al. Probing dark excitons in atomically thin semiconductors via nearfield coupling to surface plasmon polaritons. Nat. Nanotech. 12, 856 (2017).

20. Wang, G. et al. In-plane propagation of light in transition metal dichalcogenide monolayers: optical selection rules. Phys. Rev. Lett. 119, 047401 (2017).

21. Robert, C. et al. Fine structure and lifetime of dark excitons in transition metal dichalcogenide monolayers. Phys. Rev. B 96, 155423 (2017).

22. Tang, Y., Mak, K. F. \& Shan, J. Long valley lifetime of dark excitons in singlelayer WSe $\mathrm{W}_{2}$. Nat. Commun. 10, 4047 (2019).

23. You, Y. et al. Observation of biexcitons in monolayer WSe $\mathrm{W}_{2}$. Nat. Phys. 11, 477 (2015).

24. Stier, A. V. et al. Magnetooptics of exciton Rydberg states in a monolayer semiconductor. Phys. Rev. Lett. 120, 057405 (2018).

25. Barbone, M. et al. Charge-tuneable biexciton complexes in monolayer $\mathrm{WSe}_{2}$. Nat. Commun. 9, 3721 (2018).

26. Li, Z. et al. Revealing the biexciton and trion-exciton complexes in BN encapsulated $\mathrm{WSe}_{2}$. Nat. Commun. 9, 3719 (2018).

27. Chen, S.-Y., Goldstein, T., Taniguchi, T., Watanabe, K. \& Yan, J. Coulombbound four- and five-particle intervalley states in an atomically-thin semiconductor. Nat. Commun. 9, 3717 (2018).

28. Ye, Z. et al. Efficient generation of neutral and charged biexcitons in encapsulated WSe ${ }_{2}$ monolayers. Nat. Commun. 9, 3718 (2018).

29. Jones, A. M. et al. Optical generation of excitonic valley coherence in monolayer WSe2. Nat. Nanotech. 8, 634-638 (2013).

30. He, K. et al. Tightly bound excitons in monolayer WSe $\mathrm{W}_{2}$. Phys. Rev. Lett. 113 026803 (2014).

31. Ross, J. S. et al. Electrically tunable excitonic light-emitting diodes based on monolayer WSe2 p-n junctions. Nat. Nanotech. 9, 268 (2014).

32. Splendiani, A. et al. Emerging photoluminescence in monolayer $\mathrm{MoS}_{2}$. Nano Lett. 10, 1271-1275 (2010)

33. Mak, K. F., Lee, C., Hone, J., Shan, J. \& Heinz, T. F. Atomically thin $\operatorname{MoS}_{2}$ : a new direct-gap semiconductor. Phys. Rev. Lett. 105, 136805 (2010).

34. Park, K.-D., Jiang, T., Clark, G., Xu, X. \& Raschke, M. B. Radiative control of dark excitons at room temperature by nano-optical antenna-tip Purcell effect. Nat. Nanotech. 13, 59-64 (2018).

35. Liu, E. et al. Gate tunable dark trions in monolayer WSe $\mathrm{W}_{2}$. Phys. Rev. Lett. 123, 027401 (2019).

36. Aivazian, G. et al. Magnetic control of valley pseudospin in monolayer $\mathrm{WSe}_{2}$. Nat. Phys. 11, 148 (2015).

37. Srivastava, A. et al. Valley Zeeman effect in elementary optical excitations of monolayer WSe $\mathrm{W}_{2}$. Nat. Phys. 11, 141 (2015).

38. Lyons, T. P. et al. The valley Zeeman effect in inter- and intra-valley trions in monolayer $\mathrm{WSe}_{2}$. Nat. Commun. 10, 2330 (2019).

39. Li, Y. et al. Valley splitting and polarization by the Zeeman effect in monolayer $\mathrm{MoSe}_{2}$. Phys. Rev. Lett. 113, 266804 (2014).

40. MacNeill, D. et al. Breaking of valley degeneracy by magnetic field in monolayer $\mathrm{MoSe}_{2}$. Phys. Rev. Lett. 114, 037401 (2015).

41. Yu, H., Liu, G.-B., Gong, P., Xu, X. \& Yao, W. Dirac cones and Dirac saddle points of bright excitons in monolayer transition metal dichalcogenides. Nat. Commun. 5, 3876 (2014)

42. Karaiskaj, D. et al. Photoluminescence of isotopically purified silicon: how sharp are bound exciton transitions? Phys. Rev. Lett. 86, 6010-6013 (2001)
43. Wang, L. et al. One-dimensional electrical contact to a two-dimensional material. Science 342, 614-617 (2013).

\section{Acknowledgements}

This work was mainly supported by the Department of Energy, Basic Energy Sciences, Materials Sciences and Engineering Division (DE-SC0018171). Part of understanding of the valley phonon physics is supported by DoE BES DE-SC0014349. Device fabrication and part of magneto optical spectroscopy work are supported by Army Research Office (ARO) Multidisciplinary University Research Initiative (MURI) program, Grant no. W911NF-18-1-0431and NSF MRSEC 1719797. W.Y. and H.Y. were supported by Research Grants Council of Hong Kong (17312916), and Seed Funding for Strategic Interdisciplinary Research Scheme of HKU. D.G.M. and J.Y. were supported by the US Department of Energy, Office of Science, Basic Energy Sciences, Materials Sciences and Engineering Division. K.W. and T.T. were supported by the Elemental Strategy Initiative conducted by the MEXT, Japan and and the CREST (JPMJCR15F3), JST. X.X. acknowledges the support from the State of Washington funded Clean Energy Institute and from the Boeing Distinguished Professorship in Physics.

\section{Author contributions}

M.H. and P.R. fabricated the devices and performed the measurements, assisted by N.P.W and supervised by X.X. and W.Y. H.D., W.Y., D.V.T., M.Y., H.Y., M.H., P.R. and X.X. analyzed and interpreted the results. J.Y. and D.G.M. synthesized and characterized the bulk WSe ${ }_{2}$ crystals. K.W. and T.T. provided the bulk hBN crystals. M.H., P.R., X.X., H.D and W.Y. wrote the paper with inputs from all authors. All authors discussed the results.

\section{Competing interests}

The authors declare no competing interests.

\section{Additional information}

Supplementary information is available for this paper at https://doi.org/10.1038/s41467 020-14472-0.

Correspondence and requests for materials should be addressed to H.D., W.Y. or X.X.

Peer review information Nature Communications thanks the anonymous reviewer(s) for their contribution to the peer review of this work.

Reprints and permission information is available at http://www.nature.com/reprints

Publisher's note Springer Nature remains neutral with regard to jurisdictional claims in published maps and institutional affiliations.

Open Access This article is licensed under a Creative Commons Attribution 4.0 International License, which permits use, sharing, adaptation, distribution and reproduction in any medium or format, as long as you give appropriate credit to the original author(s) and the source, provide a link to the Creative Commons license, and indicate if changes were made. The images or other third party material in this article are included in the article's Creative Commons license, unless indicated otherwise in a credit line to the material. If material is not included in the article's Creative Commons license and your intended use is not permitted by statutory regulation or exceeds the permitted use, you will need to obtain permission directly from the copyright holder. To view a copy of this license, visit http://creativecommons.org/ licenses/by/4.0/

(C) The Author(s) 2020 